\section{Inkontinenz mindert die Lust bei Frauen}

\author{
Ein Harninkontinenzleiden hat Einfluss auf die Sexualfunktion und das \\ Wohlbefinden einer Frau. Eine italienische Untersuchung hat jetzt ergeben, \\ dass Frauen mit Inkontinenz vom Drang- oder Mischtyp ganz besonders \\ unter dem unfreiwilligen Urinabgang leiden.
}

$\mathrm{H}^{2}$ arninkontinenz ist ein Leiden, das viele betrifft, aber nur wenige sprechen darüber. Im Rahmen einer Beobachtungsstudie haben Salvatore Caruso und Kollegen von der Gynäkologischen Poliklinik der Universität Catania, Italien, 93 Frauen mit Harninkontinenz untersucht. Eingeschlossen wurden Patientinnen, die seit über sechs Monaten in einer stabilen heterosexuellen Partnerschaft lebten und im vorherigen Monat mindestens eine sexuelle Aktivität $\mathrm{zu}$ Protokoll gegeben hatten.

Die urodynamischen Tests hatten bei 34,5\% der Patientinnen eine Stress- bzw. Belastungsinkontinenz ergeben. Die Frauen verloren etwa beim Sport, beim Husten oder Niesen unfreiwillig Urin. Bei 37,6\% wurde eine Dranginkontinenz und bei 27,9\% ein Mischtyp festgestellt. Die Studienteilnehmerinnen erlebten mindestens eine Inkontinenzsituation pro Tag und ihr Leiden erreichte auf einer Skala von 0-10 einen Schweregrad von $\geq 5$. Die Inkontinenzdiagnose wurde mithilfe von Urinkulturen, urodynamischen Messungen, Pad- und StressTests gesichert. Frauen, die im „Pelvic Organ Prolapse Quantification“ (POPQ) auffielen, Antihypertensiva einnahmen oder Partner hatten, die unter erektiler Dysfunktion litten, waren von der Studie ausgeschlossen. Mithilfe des SF36-Fragebogens wurde die Lebensqualität überprüft und mittels Female Sexual Function Index (FSFI Gesamt-Score 2-36 aus 6 Domänen mit einer Bewertung von 0 oder 1 bis 5) und Female Sexual Distress Scale (FSDS, Score positiv ab 11 bis max. 48) Sexualfunktionen bzw. sexuelle Probleme eruiert.

\section{Stärkste Belastung bei Inkontinenz vom Mischtyp}

In allen drei Gruppen fanden sich niedrige Durchschnittswerte für die Sexualfunktionen sowie hohe bei den entsprechenden Problemen. So kamen Erre-

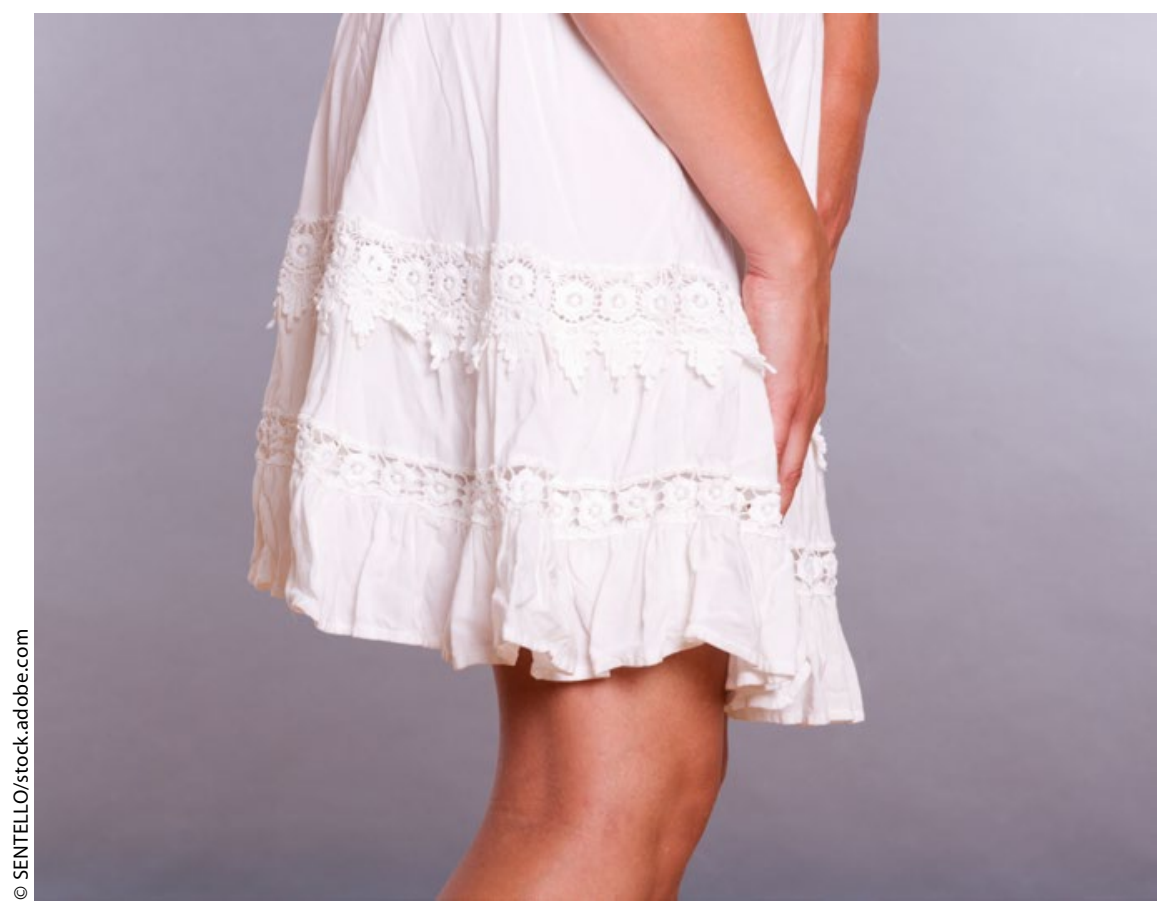

gung und Lubrikation beispielsweise nur auf Score-Werte von 2,1-2,2 bzw. 2,1-2,4. Sexuell am lebendigsten waren mit 2,8 Aktivitäten pro Monat noch Patientinnen mit Dranginkontinenz. Noch mehr negativen Einfluss auf die Frequenz hatten offenbar die Stressinkontinenz (1,2 $\mathrm{x}$ /Monat) sowie die Mischtyp-Inkontinenz (1,1 x/Monat). Diese Frauen berichteten hauptsächlich über Urinabgang während der vaginalen Penetration (93,7\% bzw. 88,5\%). Auch beim Orgasmus verloren die Patientinnen Harn, vor allem diejenigen mit Inkontinenz vom Drang- oder Mischtyp ( $94,2 \%$ bzw. 92,3\%). Allen Frauen war der unfreiwillige Urinabgang während der sexuellen Aktivitäten unangenehm.

In allen Gruppen bremste die Inkontinenz die Lust am Sex, am stärksten bei Frauen mit Mischtyp- oder Stressinkontinenz (FSFI-Score 1,1 bzw. 1,2 vs. 2,4 bei Dranginkontinenz). Auch Orgasmusstörungen traten am häufigsten bei Frauen mit Mischtyp-Inkontinenz auf, aber auch bei Dranginkontinenz (FSFI-Score: 1,4 bzw. 1,5 vs. 2,1 bei Stressinkontinenz). Insgesamt war die Sexualfunktion in der Gruppe mit Mischtyp-Inkontinenz am stärksten beeinträchtigt (FSDS-Score 28,2 vs. 24,7 bei Dranginkontinenz vs. 20,1 bei Stressinkontinenz). Frauen dieser Gruppe fanden letztlich auch die geringste sexuelle Befriedigung.

Die allgemeine Lebensqualität litt bei allen Studienteilnehmerinnen, ganz besonders bei denjenigen mit einer Inkontinenz vom Drang- oder Mischtyp (SF36: 41,5 bzw. 33,0 vs. 53,9 bei Stressinkontinenz).

$\mathrm{Da}$ urogynäkologische Störungen häufig mit sexuellen Problemen verknüpft seien, so Caruso und Kollegen, sollten bei Frauen mit Symptomen des Harntrakts generell auch die Sexualfunktion sowie persönlichen Belastungen in die Beratung einbezogen werden. Stress-, Drang- und Mischinkontinenz beeinflussten die Sexualfunktion einer Frau auf unterschiedliche Weise. Doch alle Subtypen könnten die Lebensqualität der Patientinnen erheblich verringern, so die Gynäkologen.

(cs)

Caruso $S$ et al. Effects of Urinary Incontinence Subtypes on Women's Sexual Function and Quality of Life. Urology 2017, online 24. Juni; doi: 10.1016/j.urology.2017.06.025 